\title{
Minimum Support Interpolators with Optimum Approximation Properties
}

\author{
Thierry Blu, Philippe Thévenaz and Michael Unser \\ Biomedical Imaging Group \\ EPFL-Swiss Federal Institute of Technology \\ CH-1015 Lausanne, SWITZERLAND \\ e-mail: \{thierry.blu,philippe.thevenaz,michael.unser oepfl.ch
}

\begin{abstract}
We investigate the functions of given approximation order $L$ that have the smallest support. Those are shown to be linear combinations of the Bspline of degree $L-1$ and its $L-1$ first derivatives. We then show how to find the functions that minimize the asymptotic approximation constant among this finite dimension space; in particular, a tractable induction relation is worked out. Using these functions instead of splines, we observe that the approximation error is dramatically reduced, not only in the limit when the sampling step tends to zero, but also for higher values up to the Shannon rate. Finally, we show that those optimal functions satisfy a scaling equation, althuugh less sinple than the usual two-scale difference equation.
\end{abstract}

\section{INTRODUCTION}

Most interpolation formulæ used in image processing are of the form

$$
\int_{\text {approx }}(x)=\sum_{k} c(k) \varphi\left(\frac{x}{T}-k\right)
$$

where $T$ is the sampling step and $\varphi$ the generatingor interpolating-function (e.g., a B-spline or a sinc function). The $c(k)$ 's are expansion coefficients, chosen such that $f_{\text {approx }}(x)$ is a good approximation of some hypothetical function $f(x)$ that ideally represents our data, Typically, these coefficients are obtained via a prefiltering/sampling operator acting linearly on $f$

$$
c(k)=S_{k}(f)=\int f(x) \tilde{\varphi}\left(\frac{x}{T}-k\right) \frac{d x}{T}
$$

which involves the rescaled sampling function $\tilde{\varphi}(x)$. In the simplest case (interpolation), $\tilde{\varphi}(x)$ is a linear combination of shifted Dirac masses $\delta(x-n)$ [1]. Now, when one is implementing a geometric transformation of an image, the expensive step is not so much in the determination of the $c(k)^{\prime} s$-where some very efficient filtering algorithms are available-, but rather in the computation of the expansion formula (1). Thus, the computational complexity is essentially $O\left(\left(N_{\varphi}+1\right)^{p}\right)$ per pixel, where $N_{\varphi}$ is the size of the support of $\varphi(x)$ and $p$ the number of dimensions [2]. Interestingly, the quality of the approximation (1) of $f(x)$ does not depend so much on $N_{\varphi}$, but rather on the order $L$ of the interpolator which is defined below. In return, $N_{\varphi}$ determines the computational cost. Thus, it makes sense to search for the shortest possible functions that give the order of approximation $L$ : this will provide the most efficient interpolation algorithms for a given standard of quality. Since several functions satisfy this minimum support condition, we will go one step further and optimize the interpolator so as to obtain the best image quality for a given complexity.

\section{APPROXIMATION ERROR}

In this paper, we assume that $f_{\text {approx }}(x)$ is the best (minimum error) approximation of $f(x)$ within the subspace $\operatorname{span}_{n \in \mathbb{Z}}\left\{\varphi\left(\frac{t}{T}-n\right)\right\}$, which corresponds to taking $\tilde{\varphi}(x)$ in (2) to be the dual $\varphi_{d}(x)$ of $\varphi(x)$ [3]. This optimal sampling function is best characterized in the Fourier domain

$$
\hat{\varphi}_{d}(\omega)=\frac{\hat{\varphi}(\omega)}{\sum_{n}|\hat{\varphi}(\omega+2 n \pi)|^{2}}
$$

where "^" denotes the Fourier transform operator, i.e., $\hat{\varphi}(\omega)=\int \varphi(x) e^{-i \omega x} d x$. In this paper, we thus consider the $\mathrm{L}^{2}$ approximation error $\varepsilon_{T}(f)=\left\|f-f_{\text {approx }}\right\|_{L^{2}}$ between $f$ and its approximated version.

\section{A. Approximation order}

Depending on the generating function $\varphi, \varepsilon_{T}(f)$ tends more or less rapidly to zero as $T \rightarrow 0$; the approximation scheme, or the generating function $\varphi(x)$, is thus said to be of order $L$ if $\varepsilon_{T}(f) \propto T^{L}$ as $T$ tends to zero. While this order is always considered to be integer in approximation theory, we recently constructed functions that actually have a non-integer order. We shall present them in a forthcoming paper.

Strang and Fix [4] gave a necessary and sufficient condition (under adequate hypotheses, such as compact support) for $\varphi(x)$ to be of order $L$, namcly $\hat{\varphi}(0) \neq 0$ and $\hat{\varphi}^{(l)}(2 n \pi)=0$ for all $n \in \mathbb{Z}$ and $l=0 \ldots L-1$. Unser [5] showed how to compute the proportionality constant $C_{\varphi}^{-}$between $\varepsilon_{T}(f)$ and $T^{L}$ in the limit when $T \rightarrow 0$. The exact formula

$$
C_{\varphi}^{-}=\sqrt{\sum_{n \neq 0}\left|\hat{\varphi}^{(L)}(2 n \pi)\right|^{2}}
$$

will be needed in the next section, since this is precisely the quantity that we will minimize.

\section{B. Quantitative study}

The approximation error has been studied in a more quantitative way in [6], [7], [8], [9], showing that the quality of the approximation is fully characterized by a Fourier kernel which simplifies here to

$$
E(\omega)=\frac{1}{2 \pi}\left(1-\frac{|\hat{\varphi}(\omega)|^{2}}{\sum_{n}|\hat{\varphi}(\omega+2 n \pi)|^{2}}\right) .
$$

In particular, if $f$ satisfies Shannon's sampling conditions, i.e., if the support of $\hat{f}(\omega)$ is contained within $\left[-\frac{\pi}{T}, \frac{\pi}{T}\right]$, then one has the exact relation (see also [10])

$$
\varepsilon_{T}(f)^{2}=\int|\hat{f}(\omega)|^{2} E(\omega T) d \omega
$$


What is more remarkable is that the above equation also gives the average error over all possible shifts for an arbirary function $f$, not necessarily bandlimited (c. [6], Thm.2). We recently showed the relevance of this approximation kernel as regards the computation of asymptotic expansions, upper bounds and shiftvariance estimates [8], [9].

\section{Minimum SUPPORT FUNCTIONS}

First, we give the following result which provides an explicit characterization of the shortest generating function of order $L$ [11].

Theorem 1: Minimizing for the support of the function $\varphi(x)$ under the $L^{\text {th }}$ order constraint yields piecewise polynomials that can be deduced from the B-spline function $B_{L}(x)$ of order $L$ by

$$
\varphi(x)=\sum_{k=0}^{L-1} p_{k} \frac{d^{k}}{d x^{k}} B_{L}(x)
$$

i.e., $\hat{\varphi}(\omega)=P(i \omega)\left[\frac{1-e^{-i \omega}}{i \omega}\right]^{L}$, where the polynomial $P(X)$ is defined as $P(X)=\sum_{k=0}^{L-1} p_{k} X^{k}$.

This implies, among other things, that the widely used B-spline of degree $n=L-1$ has minimum support. It is the smoothest function that has this property; it is also the only one that satisfies the standard two-scale relation and easily leads to wavelet constructions. However, (7) describes a larger class of possible interpolation functions which have not been uncovered before. In particular, this suggests that we can select the coefficients $p_{k}$ in $(7)$ such that $\varphi(x)$ satisfies some special properties. Also note that $\varphi(x)$ is continuous if $p_{L-1}=0$.

\section{A. Minimizing the approximation error}

We will now show that it is possible to outperform the B-splines in terms of the approximation error, by an appropriate choice of $P$. For this purpose, we use the explicil expression of the asymptutic constant $C_{\varphi}^{-}$(4). Replacing $\hat{\varphi}$ by its value found in (7), we get

$$
C_{\varphi}^{-}=\frac{1}{|P(0)|} \sqrt{\sum_{n \neq 0}\left|\frac{P(2 i \pi n)}{(2 i \pi n)^{t_{i}}}\right|^{2}} .
$$

$\left|C_{\varphi}^{-}\right|^{2}$ is a quadratic function of the coefficients of $P / P(0)$, i.e., it can be written as $\left|C_{\varphi}^{-}\right|^{2}=\overline{\mathrm{P}}^{\mathrm{t}} \mathrm{QP}$ where $\mathrm{P}$ and $\mathrm{Q}$ are a column vector and a matrix given by $\mathrm{P}=\left[1, p_{1} / p_{0}, \ldots p_{L-1} / p_{0}\right]^{\mathrm{t}}$ and $\mathbf{Q}_{k, l}=$ $(-1)^{k} \sum_{n \neq 0}(2 i \pi n)^{k+l-L}$, respectively.

The minimization problem is thus easily solved for P. The resulting polynomial $P_{L}(z)$ is necessarily even; thus, the optimal function $\varphi_{L}(x)$ is continuous when $L$ is even, discontinuous with bounded jumps otherwise. In practice, the linear system of equations to be solved, tends to be ill-conditioned as $L$ increases. We have thus derived an induction equation which obviates this problem. We will denote by $C_{L}$ the asymptotic constant for $\varphi_{L}$, a shorter form for $C_{\varphi_{L}}^{-}$.

Theorem 2: We have the following induction relation

$$
P_{L+1}(X)=P_{L}(X)+\frac{C_{L}^{2}}{C_{L-1}^{2}} X^{2} P_{L-1}(X) .
$$

The recursion is triggered by $P_{1}(z)=P_{2}(z)=1$.

This theorem makes it easy to compute the optimal polynomial $P_{L}$, which in turn provides the optimal function $\varphi_{L}(x)$ through (7). For example, we find

$$
\begin{aligned}
& \varphi_{4}(x)=B_{4}+\frac{1}{42} B_{4}^{\prime \prime}, \\
& \varphi_{0}(x)=B_{6}+\frac{1}{33} B_{6}^{\prime \prime}+\frac{1}{7920} B_{6}^{\prime \prime \prime \prime} .
\end{aligned}
$$

\section{B. Sampling gain}

Using these minimal functions instead of B-splines brings a non-negligible gain as shown in Table I. For example, this means that we may reduce the sampling rate in the asymptotic regime, by a factor 1.463 for $L=4$, if we use our new functions instead of the $\mathrm{B}$ splines, and still maintain the same approximation error. In other words, only two-third of the samples are sufficient if we choose the optimal approximator of order 4; moreover, this gain increases linearly with the order. Needless to say, the gain is even larger if we use approximating functions that have been shown to have much worse approximating constants than splines, such as Daubechies scaling functions (see [7], [9]).

, The optimal fourth order function is plotted in Fig. 1 where it is compared to the cubic B-spline. Moreover, the plot in Fig. 2 of the ratio between the kernel $E(\omega)$ corresponding to the optimal function and to the equivalent $\mathrm{B}$-spline, shows that the approximation using $\varphi_{4}(x)$ is always better than using $B_{4}(x)$. The gain in approximation error even exceeds $6 \mathrm{~dB}$ over half of the sampling bandwidth. According to (6), using the optimal function instead of a cubic spline for reconstruction (here $T=1$ ), we expect at least a $6 \mathrm{~dB}$ SNR gain for signals whose frequency content lays essentially in the first half of frequency domain. Obviously, this gain increases dramatically when the signal is more low-pass.

Note that our optimal functions are at most continuous. Thus, they provide a counterexample to the intuitive conjecture [5] that the most regular functions should have the smallest asymptotic approximation constant $C_{\varphi}^{-}$.

\section{Application to image processing}

These results may be of special relevance for image processing. We will see that we can obtain visible improvements over cubic spline interpolation, the state-ofthe-art method in this area [2], by using these optimized functions. This is of practical relevance since there is no penalty from the point of view of computational cost. The algorithms are essentially equivalent: the functions are cubic polynomials, have the same support, and the recursive prefilters have the same degree.

To demonstrate this fact experimentally, we used a circular symmetric test image that exhibits increasingly high frequencies near the center (see Fig. 3a). This image was rotated 16 times by a $\frac{\pi}{8}$ angle, using different interpolators; the final cumulative results are shown in Fig. 3b-e. Clearly bilinear interpolation, which is the fastest but has only two approximation orders, produces the worst results. The improvement of cubic splines over Keys interpolation algorithm, which is cubic as well, can be explained by the fact that the cubic splines have one more order of approximation, 
$L=4$ [1], [2]. The best results are obtained using our new optimized functions (Fig. 3e). The important practical point is that this improvement came to no additional cost: the algorithms in Figs c,d,e have all the same computational complexity (they all use cubic kernels of the same size).

\section{Scaling relation}

It should be added that, due to the nice multiresolution properties of B-splines, the new functions described in this paper also follow a multi-scale difference equation. For instance, if $L=4$, there is a linear relation relating $\varphi_{4}$ at scales 1,2 and 4 :

$$
\varphi_{1}(x)=\sum_{k} g_{k} \varphi_{1}(2 x-k)+\sum_{k} h_{k} \varphi_{4}(4 x-k)
$$

where the frequency transfer functions of $g_{k}$ and $h_{k}$ are $G(z)=\frac{5}{8}(1+z)^{4}$ and $H(z)=-\frac{1}{16}\left(1+z+z^{2}+z^{3}\right)^{4}$.

\section{Discussion and CONClusion}

We have presented a new family of functions that have optimal approximation characteristics. They are the smallest-support functions that have a given approximation order, and that minimize the asymptotic constant $C_{\varphi}^{-}$. We showed theoretically that the gain brought by using these functions instead of splines is very large for small sampling steps; moreover, for larger steps, the gain remains significant, as shown by Fig. 2 . We also provided an image processing example that demonstrates the improved performance of the new method. What makes this kind of optimization worthwhile is that it does not result in any additional computational cost.

\section{REFERENCES}

[1] M. Unser and 1. Daubechies, "On the approximation power of convolution-based least-squares versus interpolation," IEEE Trans. Sig. Proc., vol. 45, no. 7, pp. 1697-1711, July 1997.

[2] M. Unser, P. Thévenaz, and L. Yaroslavsky, "Convolution-based interpolation for fast, high-quality rotation of images," IEEE Trans. Imag. Proc., val. 4, no. 10, pp. 1371-1381, October 1905

[3] A. Aldroubi and $M$. Unser, "Sampling procedures in function spaces and asymptotic equivalence with Shannon's sampliag theory," Num. Funct. Anal. Opt., vol. 15, no. 1-2, pp. 1.21, February 1994

[4] G. Strang and G. Fix, "A Fourier analysis of the finite element variational method," in Constructive Aspcct of Functional Analysis, Cremonese, Ed., pp. 796-830. Rome, 1971.

[5] M. Unser, "Approximation power of biorthogonal wavelet expanjions," IEEE Trans. Sig. Proc,, vol. 44, no. 3, pp. 519-527, March 1996.

[6] T. Blu and M. Unser, "Approximation error for quasiinterpolators and (multi-) wavclet expanzions," Appl. and Comp Harm. Anal., 1997, To be published.

[7] $T$. Blu and $M$. Unser, "Quantitative $L^{2}$ error analysis for interpolation methodo and wavelet expansions," in Proc. Int. Conf. Image Processing, Santa Barbara, CA, October 1997, vol. I, pp. 663-666.

[B] T. Blu and M. Unser, "Quantitative Fouriex analysis of approximation techniques: part I-interpolators and projectors," IEEE Trans. Sig. Proc., 1998, submitted.

[9] T. Blu and M. Unzer, "Quantitative Fourier analysis of approximation techniques: part II-wavelets," IEEE Trans. Sig. Proc., 1998, submitted.

[10] C. de Boor, R.A. Devore, and A. Ron, "Approximation from shift invariant subspaces of $\mathbf{L}^{2}\left(\mathbb{R}^{d}\right)$," Trans. Amer. Math. Soc. vol. 341, no. 2, pp. 787-806, February 1994.

[11] T. Blu and M. Unser, "Minimum sllpport approximators of a given order," 1998, in preparation.
TABLE I

GAIN IN THE SAMPLING DENSITY BROUGHT BY USING THE MINIMAL INTERPOLATION INSTEAD OF THE B-SPLINE EQUIVALENT

\begin{tabular}{c||ccccc}
\hline$L$ & 1 & 2 & 3 & 4 & 5 \\
\hline \hline$\left.\frac{C_{\text {spline }}^{-}}{C_{\varphi_{L}}^{-}}\right]^{\frac{1}{L}}$ & 1 & 1 & 1.223 & 1.463 & 1.707 \\
\hline & 6 & 7 & 8 & 9 & 10 \\
\hline
\end{tabular}

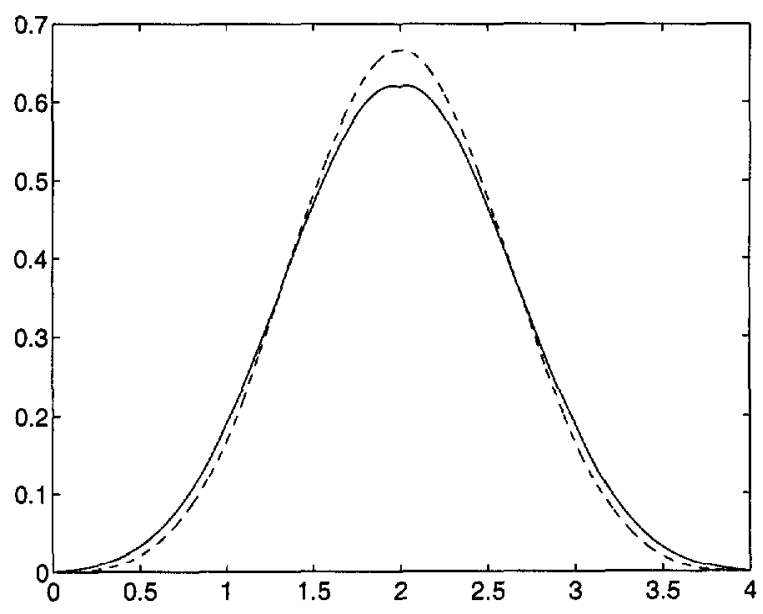

Fig. 1. Optimal function $\varphi_{\text {opt }}$ (solid) and B-spline function (dashed) for $L=4$

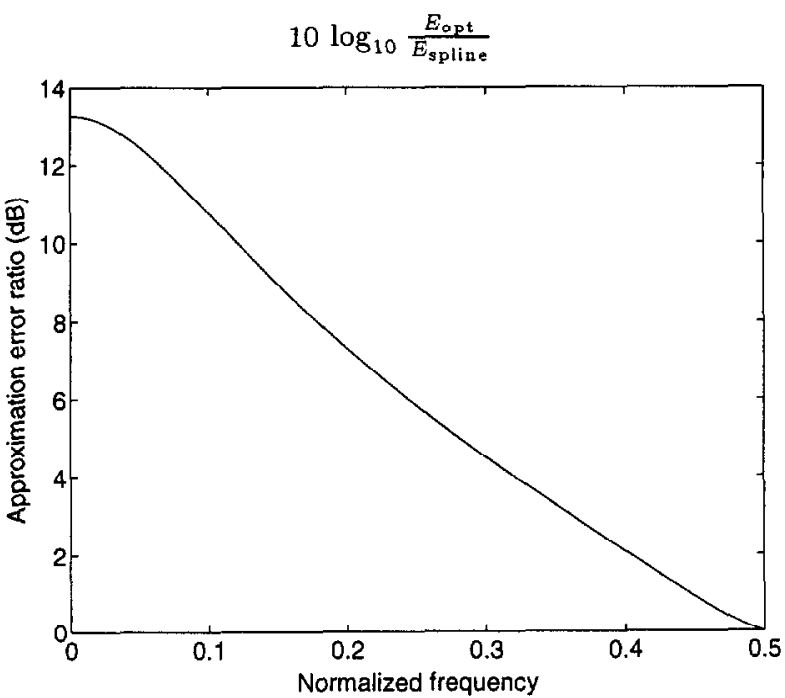

Fig. 2. Ratio between the optimal approximation kernel and the corresponding B-spline kernel for $L=4$ 


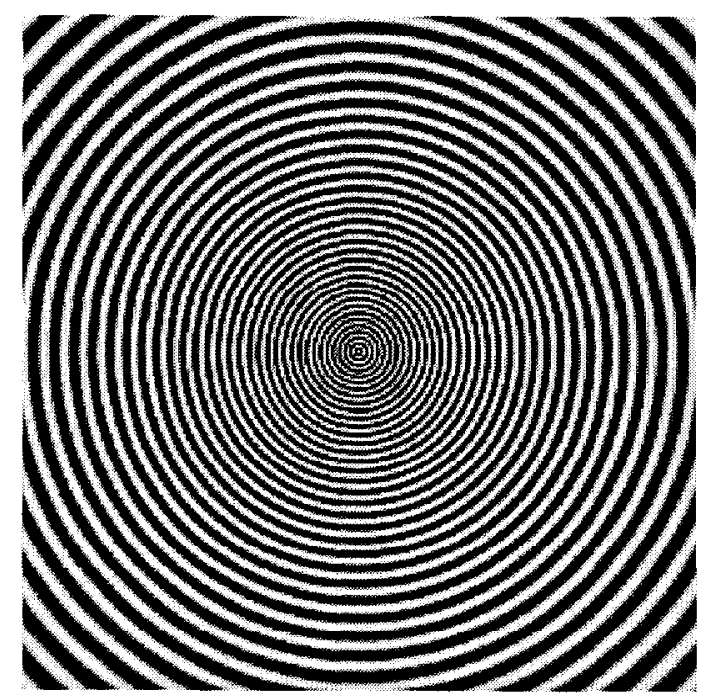

a) original image

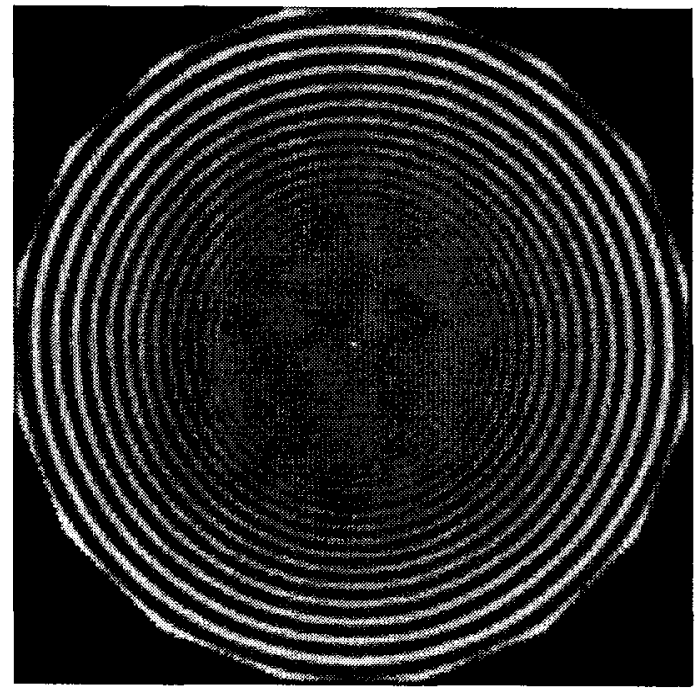

b) bilinear interpolation

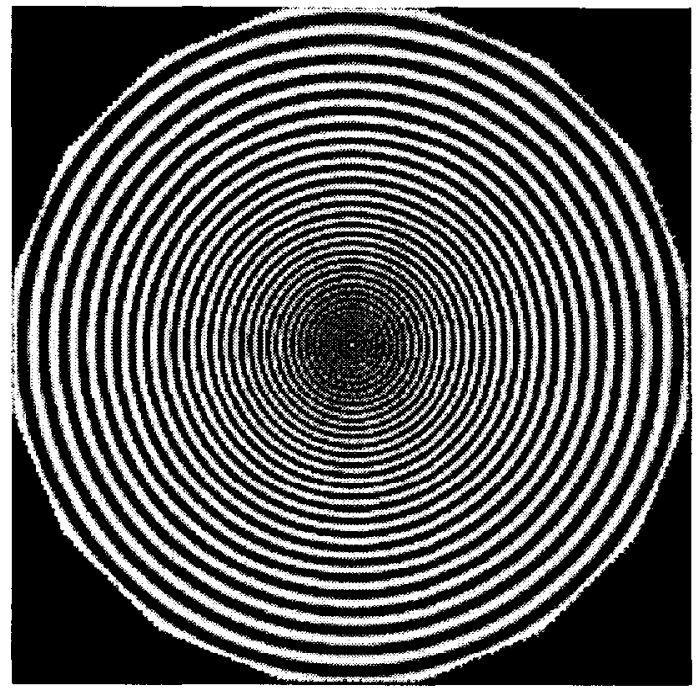

d) cubic spline interpolation

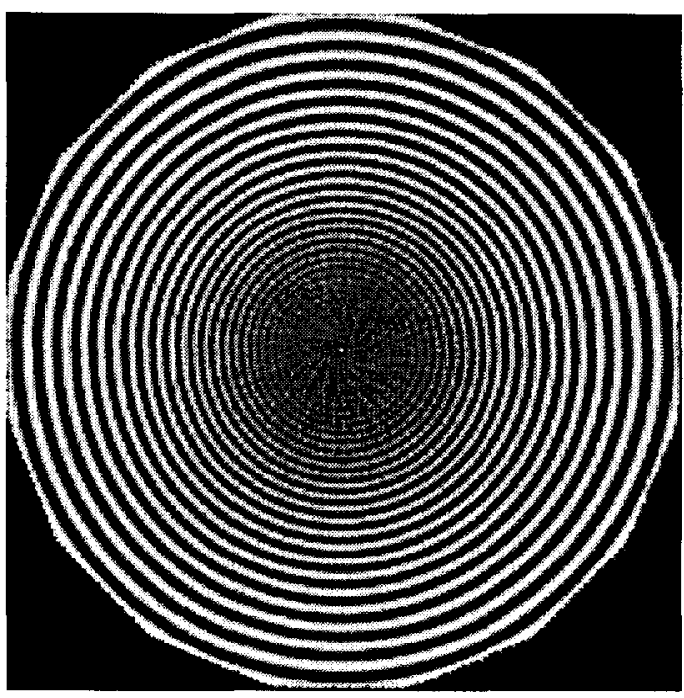

c) Keys interpolation

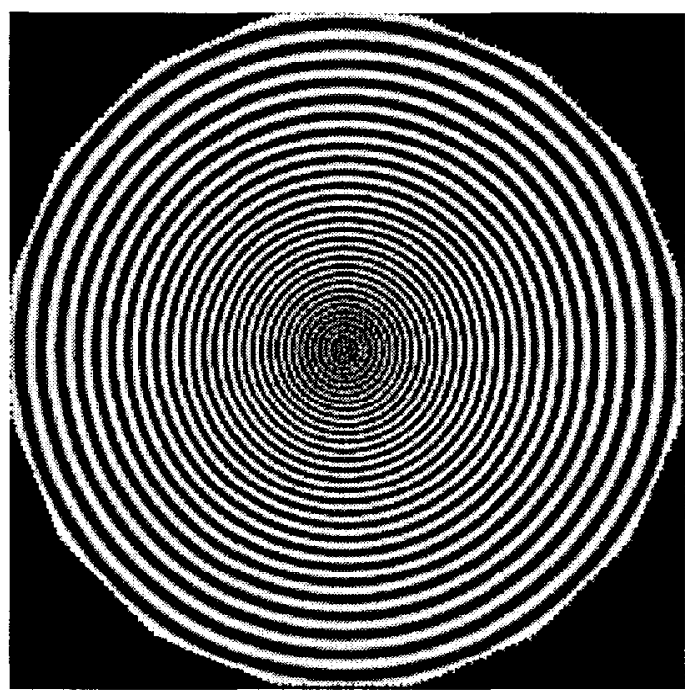

e) optimal cubic interpolation

Fig. 3. Comparison between four interpolation methods for rotating the image a (see text) 